\title{
Current landscape and trends in transdermal drug delivery systems
}

\author{
"Generic pharmaceutical marketers are increasingly keen to have a presence \\ in generic patches as they represent high-margin, high entry-barrier opportunities \\ with a smaller field of competition."
}

Keywords: drug delivery $₫$ generic patch $\|$ life cycle management $\approx$ microneedles $\|$ pain $\approx$ transdermal patch

Transdermal drug delivery systems (TDDS) have been a major contributor to the landscape of novel drug-delivery systems in the past 30 years [1]. The approval and subsequent introduction of Transderm Scop ${ }^{\circledR}$ in 1981 launched passive-delivery TDDS, commonly known as transdermal patches, as a major drug-delivery platform technology. A key scientific driver for this enduring success is the ability of TDDS to provide sustained blood levels with minimal peak-to-trough variation via a non-oral, noninjectable route of delivery. A passive-delivery patch is also the only dosage form that can bridge the dosing interval gap between a oncea-day dosing, addressed by oral dosage forms, and a once-a-week or longer dosing, which is addressed by depot injection products. These unique features and other advantageous attributes of TDDS, when matched with the right drugs, provide compelling and differentiating medical benefits to all stakeholders - patients, prescribers, payers and developers/marketers. CATAPRES-TTS $^{\circledR}$, launched in 1984, is the first patch that delivers 7-day dosing of clonidine to hypertensive patients who otherwise would have to adhere to taking one tablet twice a day [101]. This is the first example of a non-injectable sustained-release dosage form that achieves 7-day delivery for a small molecule. It demonstrates the therapeutic benefit of a dosage form that can sustain a very flat blood level profile and has the convenience of a once-a-week dosing for chronic treatment. DURAGESIC ${ }^{\circledR}$, launched in 1990, transformed post-surgical breakthrough pain care by providing a non-oral, non-injectable sustained-release opioid pain-management solution that lasts 3 days per application. DURAGESIC would go on to become the highest grossing transdermal patch with worldwide sales peaking at nearly US $\$ 2.3$ billion, before the launch of the first generic fentanyl patch in 2004 [2]. LIDODERM ${ }^{\circledR}$, a lidocaine patch launched in 1999 in the USA, introduced a new topical pain treatment option for US patients. Although originally approved for the post-herpetic neuralgia indication, its sales grew steadily as it gained additional off-label uses as a local pain treatment option, reaching over $\$ 1.1$ billion annual sales by the end of 2010 [102]. The Exelon ${ }^{\circledR}$ patch, a rivastigmine-containing patch launched in 2007, represents yet another innovative treatment option, for dementia patients and their care givers. It provides a visible reminder to both patients and their care givers that they have taken the medicine. The Exelon patch also has a superior safety profile, with, for example, less nausea and vomiting and a more convenient once-a-day dosing interval, compared with the Exelon capsule and oral solution. The Exelon patch would quickly become the main product for the Exelon franchise 3 years after its launch and prior to the loss of exclusivity of the capsule and oral solution forms [1]. Its rapid success illustrates how development of a transdermal patch can be an excellent strategy for product life cycle management.

However, not all TDDS achieved the commercial successes enjoyed by the aforementioned examples. While the topical lidocaine patch (LIDODERM) is a remarkably successful product, two other lidocaine TDDS did not fare as well. The LidoSite ${ }^{\mathrm{TM}}$ Topical System, an iontophoretic patch with a portable electronic controller and energy source, delivers lidocaine and epinephrine simultaneously by topical iontophoresis to achieve dermal analgesia on intact skin [103]. It provides dermal analgesia prior to venipunctures and other superficial dermatological procedures in $10 \mathrm{~min}$, much faster than a topical anesthetic such as EMLA (eutectic mixture of lidocaine and prilocaine) topical cream, which may take up to $60 \mathrm{~min}$ [3]. Despite its

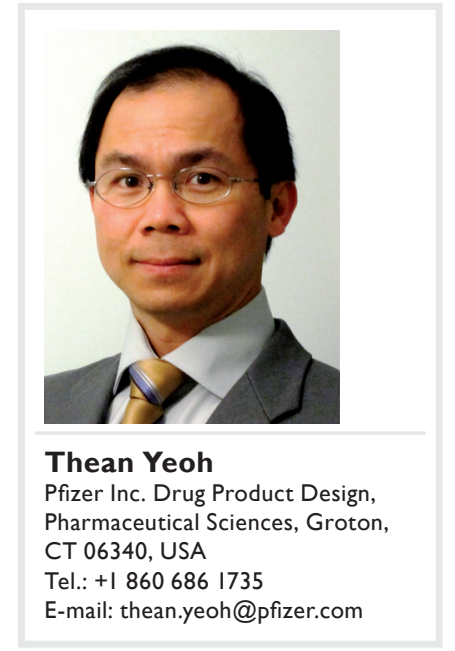

FUTURE
SCIENCE FSS 
medical advantage, for example, faster onset of action, its significantly higher treatment cost compared with a conventional topical cream limited its commercial success. Its developer and manufacturer, Vyteris, Inc., decided to withdraw Lidosite from the market in 2008 after only 2 years on the market [104]. SYNERA ${ }^{\circledR}$, a topical anesthetic patch with a controlled heating pod, delivers lidocaine and tetracaine to numb intact skin in $20 \mathrm{~min}$, prior to minor needle procedures for pediatric patients [105]. Since its launch in the USA in 2006, its uptake in the US market was disappointing [106]. While both of these products exemplify how technological advances in TDDS could foster new therapeutic advantages, for example, faster onset of action, they also make the case that TDDS need to proactively address the total value proposition to the patients and payers while providing a strong, compelling medical benefit. IONSYSTM, an iontophoretic transdermal patch for on-demand, self-activating delivery of fentanyl, allows patients to have greater control of their own pain management. In 2006, both the US FDA and the European Medicines Agency approved IONSYS for the treatment of acute post-operative pain. However, following identification of corrosion in a system component of one lot of IONSYS, which could trigger self-activation of the system - a potentially lethal overdosing risk - the European Medicines Agency recommended suspension of the marketing authorization for IONSYS in November 2008. The European Medicines Agency stated that "the company's quality systems have been unable to resolve the problems with IONSYS to prevent this quality defect [from] occurring" [107]. The European Medicines Agency also concluded that "the benefits of IONSYS no longer outweigh its risk."Janssen-Cilag, the developer and marketer of IONSYS, subsequently withdrew IONSYS from all European markets. IONSYS was very much at the cutting edge of innovation for a TDDS. Its on-demand, patientinvolved pain-management concept had the opportunity to showcase what personalized pain treatment could be. Unfortunately, high technical complexity and insurmountable product quality challenges short-circuited, literally and figuratively, this once-promising opportunity.

A recent review of product candidates in Phase I, II and III trials confirmed that the current pipeline for TDDS is both abundant and diverse [4]. However, the Phase III candidates consist mostly of passive delivery TDDS involving established small molecules already precedented for transdermal delivery. The Phase I and II candidates include a significant number of active/assisted TDDS delivering peptides and proteins, and some examples of transdermal gels. Overall, there are few examples of the traditional TDDS pipeline builders - new passive TDDS with orally delivered small molecules developed for transdermal applications.

What contributed to this cautionary trend? There is a perception, especially by the prescribers and payers, that the pharmaceutical industry is producing an increasing proportion of 'me-too' medicines; medicines, which do not show differentiating advantage over existing treatments [5]. With many safe and effective drugs already available in generic forms, successful new TDDS would have to further differentiate themselves based on enhanced safety, efficacy and convenience while also addressing cost-effectiveness. Developing new TDDS with full clinical and safety programs typically take longer and cost more, and the current industry-wide vigilance on $R \& D$ expenditure is a hindrance. Further, regulation regarding TDDS has increased in recent years as part of the regulators' efforts to improve TDDS product quality $[108,6]$.

This means that the overall hurdle for developing and introducing new TDDS has become much higher. This increasingly challenging environment also impacts the emerging active/ assisted TDDS category, which is especially sensitive to funding availability [109]. For active/ assisted TDDS technologies involving microneedles and controlled skin ablation, achieving immediate release in a less-invasive manner through the intradermal route is the key differentiating feature of the technology. This feature is especially appealing to vaccine delivery as the intradermal region of the skin is naturally rich in immuno-responsive cells [7]. For therapeutic protein and peptide delivery, while intradermal delivery may provide a more favorable pharmacokinetic profile compared with subcutaneous or intramuscular injections, other tangible patient benefits, such as easy self-administration, less perceived pain and enhanced safety, are also critical to make this a compelling product concept. However, the requirement for molecular potency is limiting the size of this opportunity to selective proteins and peptides. Conversely, only limited opportunities exist for small molecules in this area, as product differentiation would not only be measured against the conventional injectables but also the 'low-cost' oral dosage forms. The cost base for this technology will need to become 
lower to induce broader applications. In a recent positive development in this direction, microneedle therapeutics research pioneer Mark Prausnitz will pursue an innovative global health research project focused on demonstrating the scientific and economic feasibility of using microneedle patches for the low-cost administration of vaccination programs [110]. An initiative, such as this, which seeks to achieve cost-effectiveness as a goal of innovation, is beneficial to emerging TDDS technology. Favorable demonstration of cost-effectiveness could greatly expand the opportunity for TDDS technology.

With the opportunities and constraints surrounding the diverse TDDS space, what can we expect for the next 5 years? For one, we can expect the continued introduction of passivedelivery generic patches. As highly successful products such as LIDODERM and Exelon are nearing their respective loss of exclusivity, multiple generic lidocaine (for the US market) and rivastigmine patches will launch in the upcoming years. Generic pharmaceutical marketers are increasingly keen to have a presence in generic patches as they represent high-margin, high entry-barrier opportunities with a smaller field of competition. Since 2007, the FDA has published 12 bioequivalence draft guidances for generic transdermal patches [8]. This has leveled the playing field and encouraged new developers to enter this area, making generic TDDS an area of growth. For other new passive-delivery TDDS, opportunities remain for differentiated products, especially once-a-week-dosing products, which enhance convenience and compliance, and potentially treatment outcome. The emerging active/ assisted TDDS is entering a watershed period as stakeholders eagerly await the first wave of active/ assisted delivery TDDS products to obtain regulatory approval. The cost effectiveness of this technology will be an area to watch as it holds the key to either expand or limit future opportunities.

\section{Financial \& competing interests disclosure}

The author is an employee of Pfizer. The views and opinions expressed in this editorial are solely those of the author. The author has no other relevant affiliations or financial involvement with any organization or entity with a financial interest in or financial conflict with the subject matter or materials discussed in the manuscript apart from those disclosed. No writing assistance was utilized in the production of this manuscript.

\section{References}

1 Yeoh T. Profiles of recently approved transdermal drug delivery systems (TDDS). Part 1: Exelon patch - example of life cycle strategy for successful products; generic clonidine transdermal systems - design and delivery attributes. TransDermal Mag. 3(4), 10-17 (2011).

2 Yeoh T. Profiles of recently approved transdermal drug delivery systems (TDDS). Part II: Matrix-type fentanyl transdermal systems - design variation and delivery attributes. TransDermal Mag. 3(5), 9-15 (2011).

3 Zempsky WT. Reduction of pain associated with minor procedures in children - new topical anesthetics on the horizon. Touch Briefings. Business Briefing: US pediatric Care. 19-22 (2005).

$4 \quad$ Hill L. Joining the transdermal pipeline. TransDermal Mag. 3(4), 5-8 (2011).

5 Schmid EF, Smith DA. Is declining innovation in the pharmaceutical industry a myth? Drug Discov. Today 10(15), 1031-1039 (2005).

6 Guidance for industry. Residual Drug in Transdermal and Related Drug Delivery Systems. US Department of Health and Human Services Food and Drug Administration Center for Drug Evaluation and Research (CDER), August 2011.
7 Lamberta PH, Laurent PE. Intradermal vaccine delivery: will new delivery systems transform vaccine administration? Vaccine 26(26), 3197-3208 (2008).

8 Lehman PA. Analysis of new FDA draft guidances: insights on study designs for bioequivalence assessment of transdermal systems. Drug Develop. Deliv. 11(3), 36-41 (2011).

\section{Websites}

101 US prescription information for Catapres ${ }^{\circledR}$. www.drugs.com/pro/catapres.html

102 Press release from Endo Pharmaceuticals. http://phx.corporate-ir.net/phoenix. zhtml? $\mathrm{c}=123046 \& \mathrm{p}=$ irol-newsArticle $\&$ ID $=5$ 24520\&highlight=

103 Full prescription information for LidoSite ${ }^{\circledast}$ Topical System. www.vyteris.com/legal/LidositePI_ PN20137Rev1.pdf

104 Annual report of Vyteris Holdings Inc., Form 10-K. www.faqs.org/sec-filings/100325/VyterisHoldings-Nevada-Inc_10-K/

105 Full prescription information for Synera. www.synera.com

106 Quarterly report of Endo Pharmaceuticals Holdings Inc., Form 10-Q. http://sec.edgar-online.com/endopharmaceuticals-holdings-inc/10-q-quarterlyreport/2008/08/01/section7.aspx

107 European Medicines Agency press release: European Medicines Agency recommends the suspension of the marketing authorisation of Ionsys (fentanyl hydrochloride). www.ema.europa.eu/ema/index. jsp?curl=pages/news_and_events/ news/2009/11/news_detail_000249. jsp \& mid=WC0b01ac058004d5c1

108 Improved development and regulation of transdermal systems - a DIA workshop co-sponsored by the FDA. Arlington, Virginia, USA.

www.diahome.org/DIAHome/Education/ FindEducationalOffering. aspx? productID =2 7035\&event Type $=$ Meeting \&utm source $=$ DIAHomePageNA\&utm medium $=$ Button \&utm_campaign $=11021$

109 Karkaria U. \$60M later, biotech Altea calls it quits. Atlanta Business Chronicle, 9 December (2011).

www.bizjournals.com/atlanta/printedition/2011/12/09/60m-later-biotech-alteacalls-it-quits.html

110 Press release from the Georgia Institute of Technology newsroom. www.gatech.edu/newsroom/release. html?nid $=72457$ 